\title{
Measurement of intensity and polarization beatings in the interference of independent optical fields
}

\author{
Andriy Shevchenko ${ }^{*} *$ \\ Department of Applied Physics, Aalto University, P.O. Box 13500, FI-00076 Aalto, Finland \\ Tero Setälä \\ Institute of Photonics, University of Eastern Finland, P.O. Box 111, FI-80101 Joensuu, Finland
}

(Received 7 January 2020; accepted 22 February 2020; published 5 March 2020)

\begin{abstract}
We experimentally study the intensity and polarization beatings of interfering independent optical fields. Using the tools of intensity interferometry, we measure the beating harmonicity time and the degree of randomness of the polarization beating for several combinations of light fields with Poissonian and Gaussian statistics. The degree of randomness is represented by the effective thickness of a ring on the Poincaré sphere within which the tip of the Poincaré vector moves. The method is based on two-photon detection, allowing femtosecond-scale variations of light intensity and polarization to be experimentally characterized. The research contributes to both fundamental and applied aspects of electromagnetic optics.
\end{abstract}

DOI: 10.1103/PhysRevResearch.2.012053

\section{INTRODUCTION}

Polarimetric characterization of optical beams is necessary in the diversity of topics concerning classical and quantized light $[1,2]$. The traditional concepts cover the Jones vector, Stokes parameters, Poincaré sphere, Mueller matrices, and degree of polarization, for instance $[3,4]$. Concerning the highly nonparaxial fields, possibly with an evanescent-wave contribution, several novel polarimetric descriptors such as the polarimetric dimension [5], degree of intensity anisotropy [6], and nonregularity [7] have recently been introduced. Interference is another fundamental attribute of light with important applications, e.g., in optical communication and information processing [3], laser cavity design [8], correlation imaging [9], spectroscopy [10], and metrology [11]. In the context of electromagnetic (vectorial) light, interference may show up as an intensity modulation or polarization-state variation or both, a fact which has had significant implications in the description of electromagnetic coherence [12,13].

Despite extensive literature concerning both interference and polarimetry, interference of electromagnetic beams with different frequencies has not been much studied. In such a case the total field exhibits wave beating, in which both the intensity and polarization state may vary periodically as well as randomly in time. For independent light beams this effect was recently theoretically analyzed in Ref. [14]. It was shown that the beating can be considered essentially harmonic

\footnotetext{
*andriy.shevchenko@aalto.fi

Published by the American Physical Society under the terms of the Creative Commons Attribution 4.0 International license. Further distribution of this work must maintain attribution to the author(s) and the published article's title, journal citation, and DOI.
}

within a certain beating harmonicity time which depends on the beam bandwidths [14]. If the beams have different polarization states, the polarization evolution of the total field can be described by a rotating Poincaré vector that draws a randomized circular trajectory, forming with time a ring on the Poincaré sphere. The thickness of the ring was predicted to depend on the statistics that the interfering fields obey [14].

In this Rapid Communication, we experimentally study the beating effects in the interference of independent optical fields and measure the beating harmonicity time and the thickness of the polarization ring on the Poincaré sphere, using optical sources that obey Gaussian and Poissonian statistics. In the experiments, we utilize a Michelson interferometer and a semiconductor photomultiplier tube working in the twophoton absorption regime, which allows ultrafast variations of light intensity and polarization to be detected and statistically characterized [15-17]. The results of this work contain an experimental verification of the theoretical results in Ref. [14].

\section{THEORETICAL BACKGROUND}

Let two interfering monochromatic electromagnetic waves have the same polarization states, but different frequencies. The intensity of the superposition field is in this case a harmonic function of time with a period

$$
T_{\mathrm{b}}=2 \pi /\left|\omega_{1}-\omega_{2}\right|,
$$

where $\omega_{1}$ and $\omega_{2}$ are the angular frequencies of the waves $[14,17]$. If the waves also have different polarization states, then not only the intensity, but also the polarization of the total field varies with the same period $T_{\mathrm{b}}$. The polarization beating is a continuous periodic change of the polarization state that can be represented on the Poincaré sphere by a continuous rotation of the Poincaré vector whose tip traces a circular trajectory $[14,17]$. 
If the interfering fields are not monochromatic, but have some well separated spectra, the beating period can still be calculated from Eq. (1), but with $\omega_{1}$ and $\omega_{2}$ being replaced by the center frequencies of the fields. However, the beating is no longer fully predictable, because the amplitudes and phases of the interfering fields fluctuate. The beating predictability (harmonicity) can be studied by measuring the intensity autocorrelation function $\langle I(t) I(t+\tau)\rangle$ [18] of a polarization component of the total field to which both interfering waves contribute. The autocorrelation function may oscillate, but the value of its envelope decreases when the intensity correlation disappears. The function can be normalized as

$$
m(\tau)=\frac{\langle I(t) I(t+\tau)\rangle-\langle I(t)\rangle^{2}}{\left\langle I^{2}(t)\right\rangle-\langle I(t)\rangle^{2}}
$$

to obtain $m(0)=1$ and $m(\tau \rightarrow \infty)=0$. The beating harmonicity time, denoted by $\tau_{\mathrm{bh}}$, can be defined by requiring that the envelope of $m(\tau)$ decreases to $1 / 2$ at $\tau=\tau_{\text {bh }}$.

For independent fields with Gaussian spectra and Gaussian statistics, one can obtain

$$
\tau_{\mathrm{bh}} \approx \frac{2\left(\bar{I}_{1}+\bar{I}_{2}\right)}{\bar{I}_{1} \Delta \omega_{1}+\bar{I}_{2} \Delta \omega_{2}},
$$

where $\bar{I}_{1}$ and $\bar{I}_{2}$ are the average intensities and $\Delta \omega_{1}$ and $\Delta \omega_{2}$ the spectral widths [full widths at half maxima (FWHM)] of the interfering waves [14]. The beating harmonicity time can be shorter than the beating period, which at equal $\bar{I}_{1}$ and $\bar{I}_{2}$ requires that $\left|\omega_{1}-\omega_{2}\right|<\Delta \omega_{1}+\Delta \omega_{2}$. It is also remarkable that, if one of the beams is nearly monochromatic so that $\Delta \omega_{1} \ll \Delta \omega_{2}$ and we still have $\bar{I}_{1}=\bar{I}_{2}$, the beating harmonicity time is approximately equal to the coherence time of the beam with the wider spectrum. This result is explained by the fact that the phase and amplitude fluctuations of the quasimonochromatic component are slow, and the beating deviates from harmonic only because of random variations of the broadband component that, on average, take place during the field's coherence time. If the intensities considerably differ, the function $m(\tau)$ is only weakly modulated by beating and describes mostly the self-correlation of the strong field. Therefore, in order to measure purely the beating process, the intensities of the interfering components should be set equal. Although Eq. (3) is derived for fields with Gaussian statistics, the experiments show, as we will see in Sec. III, that it holds also if one or both fields obey Poissonian statistics.

The polarization beating is conveniently described in terms of the Poincaré vector $\mathbf{S}=\left(S_{1}, S_{2}, S_{3}\right)$, whose length $S_{0}$ and the three vector components are equal to the instantaneous Stokes parameters $S_{0}=\left|E_{x}\right|^{2}+\left|E_{y}\right|^{2}, S_{1}=\left|E_{x}\right|^{2}-$ $\left|E_{y}\right|^{2}, S_{2}=2 \operatorname{Re}\left\{E_{x}^{*} E_{y}\right\}$, and $S_{3}=2 \operatorname{Im}\left\{E_{x}^{*} E_{y}\right\} \quad[1,14]$. Here, $E_{x}$ and $E_{y}$ can be any two orthogonal vector components of the total electric field $\mathbf{E}$. If the beating waves are monochromatic and polarized along the $x$ and $y$ axis, the Poincaré vector $\mathbf{S}$ rotates at a constant speed in a plane perpendicular to the axis $S_{1}$ with a period given by Eq. (1). If the wave intensities are the same, the rotation plane coincides with the $S_{2} S_{3}$ plane, as shown in Fig. 1(a). If the fields are polychromatic, the vector $\mathbf{S}$ fluctuates during rotation, and the trajectory of its tip forms a torus, or a ring, with a certain effective thickness $\sigma$ on the unitradius Poincaré sphere [see Fig. 1(b)]. The Poincaré vector
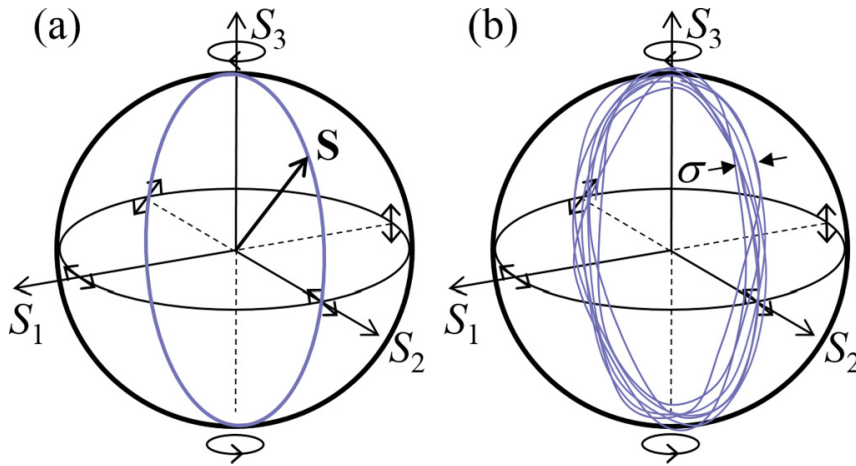

FIG. 1. Polarization beating of orthogonally polarized, equalintensity light waves with different center frequencies illustrated on the Poincaré sphere. In (a) the waves are monochromatic and in (b) polychromatic. The path of the rotating Poincaré vector in (b) is not perfectly circular, filling with time a ring area with effective thickness $\sigma$.

is in this case normalized to have a unit length by dividing the Stokes parameters with the total intensity of the field. The quantity $\sigma$ characterizes the randomness of the rotation of the Poincaré vector. It has been shown in Ref. [14] that $\sigma$ can be expressed in terms of the instantaneous intensities $I_{1}$ and $I_{2}$ of the interfering orthogonally polarized fields by calculating the rms value of the projection of the Poincare vector on the direction $\mathbf{s}_{\perp}$ perpendicular to the ring, i.e.,

$$
\sigma=\sqrt{3 \frac{\left\langle\left(\mathbf{S} \cdot \mathbf{s}_{\perp}\right)^{2}\right\rangle}{\left\langle S_{0}^{2}\right\rangle}}=\sqrt{3 \frac{\left\langle I_{1}^{2}\right\rangle+\left\langle I_{2}^{2}\right\rangle-2\left\langle I_{1} I_{2}\right\rangle}{\left\langle I_{1}^{2}\right\rangle+\left\langle I_{2}^{2}\right\rangle+2\left\langle I_{1} I_{2}\right\rangle}} .
$$

Above, the normalization factor $\sqrt{3 /\left\langle S_{0}^{2}\right\rangle}$ is introduced for the fields obeying Gaussian statistics with $\left\langle I_{1}\right\rangle=\left\langle I_{2}\right\rangle$ to have $\sigma=0$ if the fields correlate and $\sigma=1$ if they are statistically independent [14]. The maximum value of 1 implies that the orientation of the Poincaré vector is fully unpredictable, and the trajectory of its tip, with time, fills the whole Poincaré sphere.

If the fields are of the Poissonian statistics and their intensities are high, the instantaneous intensities of the interfering waves are essentially constant (the signal-to-noise ratio is high [3]) and the thickness of the ring approaches zero for both mutually correlated and independent fields. Indeed, as long as the field intensities are constant, the Poincaré vector draws a perfect circle, even though its rotation speed randomly changes in time. If, on the other hand, one of the (independent) fields obeys Gaussian statistics and the other one Poissonian statistics, the ring thickness $\sigma$ takes the value of $\sqrt{3 / 5}$ [14] Indeed, if the first field has Gaussian statistics, we obtain $\left\langle I_{1}^{2}\right\rangle=2\left\langle I_{1}\right\rangle^{2}$, and if the second field is of Poissonian statistics, we obtain $\left\langle I_{2}^{2}\right\rangle=\left\langle I_{1}\right\rangle^{2}$ and $\left\langle I_{1} I_{2}\right\rangle=\left\langle I_{1}\right\rangle\left\langle I_{2}\right\rangle$. Substituting these results into Eq. (4) and setting $\left\langle I_{1}\right\rangle=\left\langle I_{2}\right\rangle$ gives $\sigma=\sqrt{3 / 5}$.

\section{EXPERIMENTAL RESULTS}

Equations (2) and (4) are written in terms of the intensity correlation functions that can be measured, e.g., by using intensity interferometry and two-photon detection [16,17]. The experimental setup employed in this work is shown in 


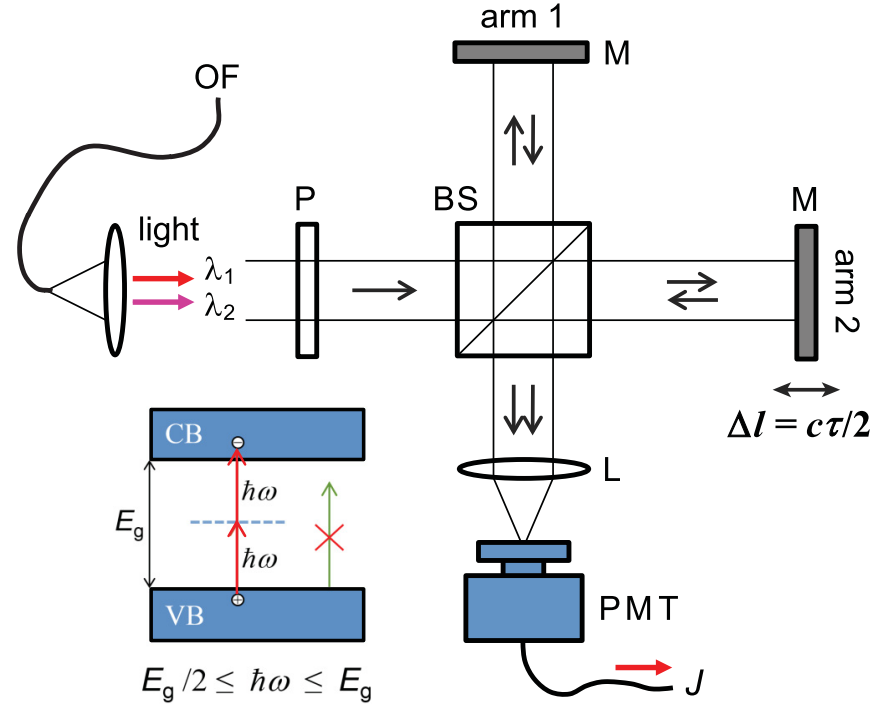

FIG. 2. Experimental setup used to measure the beating harmonicity time. OF: optical fiber; P: polarizer; BS: beam splitter; M: mirror; L: lens; and PMT: photomultiplier tube. The photomultiplier tube operates in the two-photon absorption regime as long as the light frequency $\omega$ and the detector band gap $E_{\mathrm{g}}$ satisfy the condition $E_{\mathrm{g}} / 2 \leqslant \hbar \omega \leqslant E_{\mathrm{g}}$, as shown by the inset. VB and CB stand for the valence and the conduction band, respectively.

Fig. 2. Two independent orthogonally polarized optical beams with center wavelengths $\lambda_{1}$ and $\lambda_{2}$ are combined with a polarizing beam splitter and delivered to a Michelson interferometer in a polarization-maintaining single-mode optical fiber. A polarizer $(\mathrm{P})$ is used at the input of the interferometer to enable the two beams to produce interference fringes. Its transmission axis is set to an angle at which the powers of the transmitted beams are equal. The beam splitter of the interferometer is polarization insensitive. The output beam of the interferometer is focused into a semiconductor photomultiplier tube (Hamamatsu H7421-50) with a band-gap wavelength of $900 \mathrm{~nm}$. The detector operates in the two-photon absorption regime in the spectral range of $\lambda \in[900 \mathrm{~nm}, 1800 \mathrm{~nm}]$ (see the inset of Fig. 2). The length of one interferometer arm can be tuned by moving the mirror at the arm end. The time difference $\tau$ that appears in the intensity correlation function $\langle I(t) I(t+\tau)\rangle$ of Eq. (2) is determined by the length difference $\Delta l$ of the interferometer's arms via $\tau=2 \Delta l / c$. The measured two-photon-absorption signal at each $\tau$ can be written in terms of the instantaneous intensities $I(t)$ and $I(t+\tau)$ of the beams coming from the two arms as [17]

$$
\begin{aligned}
J(\tau)= & A\left[\left\langle I^{2}(t)\right\rangle+\left\langle I^{2}(t+\tau)\right\rangle+4\langle I(t) I(t+\tau)\rangle\right] \\
& +B \cos \left(\omega_{\mathrm{av}} \tau\right)+C \cos \left(2 \omega_{\mathrm{av}} \tau\right),
\end{aligned}
$$

where $A, B$, and $C$ are proportionality coefficients whose form is not relevant. The last two rapidly oscillating terms can be filtered out by averaging, e.g., by Fourier-transforming the signal and removing the spectral powers around $\omega_{\mathrm{av}}$ and $2 \omega_{\mathrm{av}}$, where $\omega_{\mathrm{av}}=\left(\omega_{1}+\omega_{2}\right) / 2$. The first two terms are equal and independent of $\tau$. They can be measured by blocking the beam in one of the arms and measuring $J(\tau)$. The intensity correlation function $\langle I(t) I(t+\tau)\rangle$ can therefore be written in

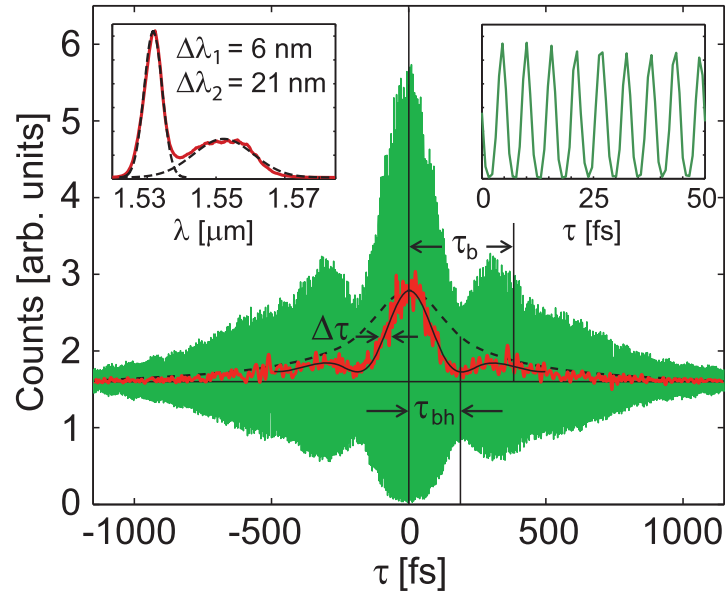

FIG. 3. The photomultiplier signal $J(\tau)$ (green line) and its filtered version (red line) measured for an amplified spontaneous emission of an Er-doped fiber. The dashed line is an approximate envelope function for the red curve. The inset in the left corner shows the light spectrum (red) that is split into two Gaussian peaks (dashed lines). The right-corner inset shows a zoomed-in part of the green curve. The quantities $\tau_{\mathrm{b}}$ and $\tau_{\mathrm{bh}}$ are the beating period and the beating harmonicity time, respectively. The black solid curve shows fitting to the red curve, as explained in the text, and $\Delta \tau=20 \mathrm{fs}$.

terms of the filtered signal $J_{\mathrm{f}}(\tau)$ as $\langle I(t) I(t+\tau)\rangle=D J_{\mathrm{f}}(\tau)+$ $F$, where $D$ and $F$ are constants.

\section{A. Beating harmonicity time}

In the first example, we use the two emission bands of an Er-doped fiber amplifier, each generating amplified spontaneous emission (ASE) obeying Gaussian statistics. Figure 3 shows the source spectrum with the two bands fitted by two dashed Gaussian curves (see the inset in the left corner). The generated ASE light can therefore be treated as a superposition of two optical fields with the center wavelengths $\lambda_{1} \approx$ $1532 \mathrm{~nm}$ and $\lambda_{2} \approx 1553 \mathrm{~nm}$ and bandwidths $\Delta \lambda_{1} \approx 6 \mathrm{~nm}$ and $\Delta \lambda_{2} \approx 21 \mathrm{~nm}\left(\Delta \omega_{1}=4.8 \mathrm{THz}\right.$ and $\left.\Delta \omega_{2}=16.4 \mathrm{THz}\right)$. The intensities of these two fields can be evaluated as $\bar{I}_{i} \approx$ $S_{0 i} \Delta \lambda_{i}$, where $S_{0 i}$ is the peak spectral power density of band $i$. These calculations give $\bar{I}_{1} / \bar{I}_{2}=24 / 22 \approx 1$ and Eq. (3) results in $\tau_{\mathrm{bh}} \approx 4 /\left(\Delta \omega_{1}+\Delta \omega_{2}\right) \approx 190 \mathrm{fs}$. The beating harmonicity time turns out to be shorter than the beating period that in accordance with Eq. (1) is $\tau_{\mathrm{b}} \approx 380 \mathrm{fs}$.

To verify these results experimentally, we coupled the ASE light to the input fiber of the interferometer in Fig. 2 and measured $J(\tau)$. The power was kept below $1 \mathrm{~mW}$ to ensure a quadratic dependence of the photomultiplier signal on the input power [17]. The result is shown in Fig. 3 by the green line, a fragment of which is shown also in the second inset for $\tau \in[0,50] \mathrm{fs}$. The red line depicts the filtered signal $J(\tau)$ that is equal to the intensity correlation function obtained from the green curve and scaled in the vertical direction by tuning $D$ and $F$. The resulting red curve crosses the center points between the neighboring maxima and minima of the rapidly oscillating green curve. The envelope of the red curve is shown by the dashed line. The measured beating harmonicity time is shown in the figure. Its value is measured with 


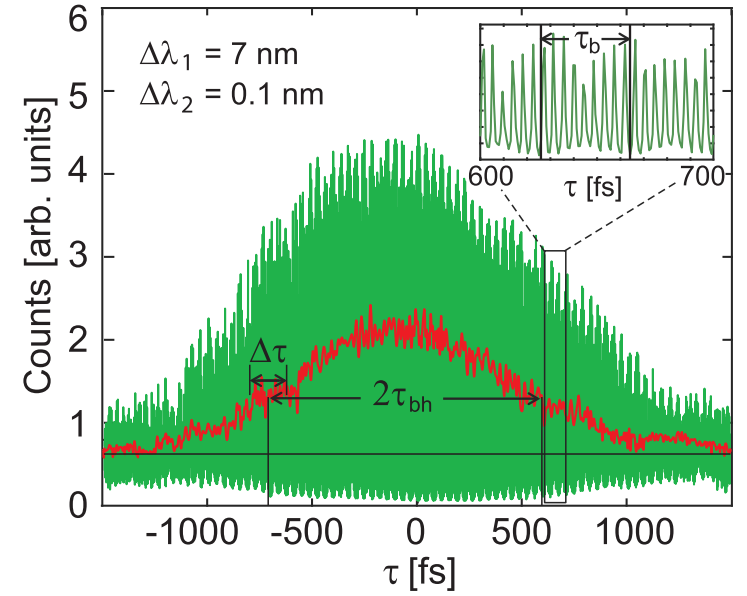

FIG. 4. The photomultiplier signal $J(\tau)$ (green line) and its filtered and scaled version (red line) measured for a field composed of interfering laser beams with the wavelengths of $\lambda_{1}=1480 \mathrm{~nm}$ and $\lambda_{2}=1310 \mathrm{~nm}$ obeying Gaussian and Poissonian statistics, respectively. Their bandwidths are $\Delta \lambda_{1}$ and $\Delta \lambda_{2}$. The inset shows a zoomed-in part of the measured curve $J(\tau)$. The quantities $\tau_{\mathrm{b}}$ and $\tau_{\mathrm{bh}}$ are the beating period and the beating harmonicity time, respectively. The width $2 \tau_{\mathrm{bh}}$ of the red curve is measured with an uncertainty of $\Delta \tau=180 \mathrm{fs}$.

an uncertainty determined by $\Delta \tau=20$ fs shown in Fig. 3 . Thus, we have $\tau_{\mathrm{bh}}=190 \pm 10$ fs. This corresponds to the value obtained above from Eq. (3). In addition, the measured beating period is $\tau_{\mathrm{b}} \approx 380 \mathrm{fs}$ consistently with the theoretical estimate. This value was obtained by fitting the red line with the curve $C_{1}+C_{2} \exp \left(-\ln 2 \tau^{2} / \tau_{\mathrm{bh}}^{2}\right) \cos ^{2}\left(\pi \tau / \tau_{\mathrm{b}}\right)$, where $C_{1}$ and $C_{2}$ are constants. The fitting curve is shown in the figure by the black solid line. The uncertainty in the measurement of $\tau_{\mathrm{b}}$ is also on the order of $10 \mathrm{fs}$. These results demonstrate good agreement between the theory and the measurements.

Then we examined interference of two orthogonally polarized beams radiated by independent laser diodes, of which one obeys Gaussian statistics (L1480G1, Thorlabs) and the other Poissonian statistics (L1310P5DFB, Thorlabs). Their center wavelengths and bandwidths are $\lambda_{1} \approx 1480 \mathrm{~nm}, \Delta \lambda_{1} \approx 7 \mathrm{~nm}$, $\lambda_{2} \approx 1310 \mathrm{~nm}$, and $\Delta \lambda_{2} \approx 0.1 \mathrm{~nm}$, respectively. In terms of angular frequencies, the bandwidths are $\Delta \omega_{1}=6.0 \mathrm{THz}$ and $\Delta \omega_{2}=0.1 \mathrm{THz}$. The powers (intensities) of the two beams were set equal. Since $\Delta \omega_{2} \ll \Delta \omega_{1}$, Eq. (3) yields $\tau_{\mathrm{bh}} \approx 4 / \Delta \omega_{1} \approx 667 \mathrm{fs}$. The beating harmonicity time is now much longer than the beating period $\tau_{\mathrm{b}} \approx 38 \mathrm{fs}$. The measured signal (green curve) and the intensity correlation function (red curve) are shown in Fig. 4. The inset shows a zoomed part of the green curve from which the beating period of $38 \mathrm{fs}$ can be seen. The measured beating harmonicity time is $\tau_{\mathrm{bh}}=660 \pm 90 \mathrm{fs}$ corresponding to the one calculated above. Again the theoretical results match well to the experimental ones. In addition, they suggest that Eq. (3) is accurate also for fields obeying Poissonian statistics.

In order to confirm the validity of Eq. (3) for singlefrequency laser beams both obeying Poissonian statistics, we replaced the first laser diode (L1489G1) with a singlefrequency one radiating at $\lambda_{1}=1550 \mathrm{~nm}$ (L1550P5DFB, Thorlabs). Its bandwidth is $\Delta \lambda_{1} \approx 0.1 \mathrm{~nm}\left(\Delta \omega_{1}=78 \mathrm{GHz}\right)$.

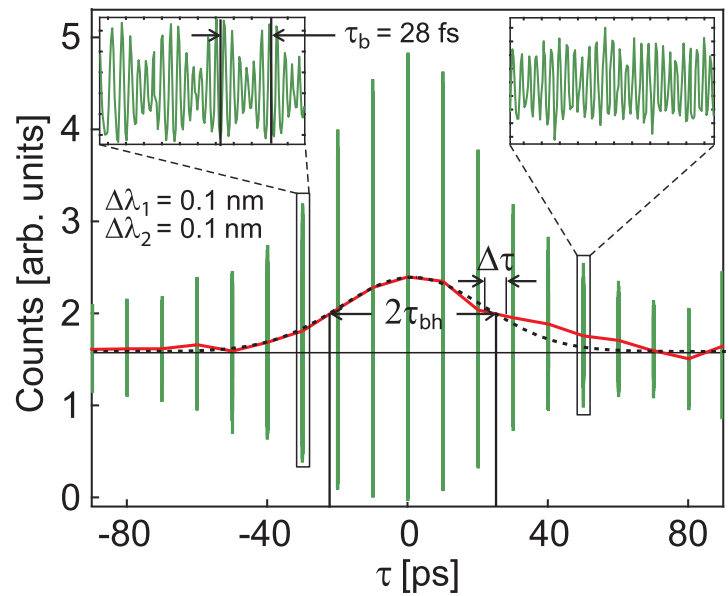

FIG. 5. The photomultiplier signal $J(\tau)$ (green line) and its locally time-averaged version (red line) measured piecewise for a field composed of interfering laser beams with the wavelengths of $\lambda_{1}=1550 \mathrm{~nm}$ and $\lambda_{2}=1310 \mathrm{~nm}$, both obeying Poissonian statistics. Their bandwidths are $\Delta \lambda_{1} \approx \Delta \lambda_{2} \approx 0.1 \mathrm{~nm}$. The insets show two zoomed-in parts of the measured curve $J(\tau)$. The quantities $\tau_{\mathrm{b}}$ and $\tau_{\mathrm{bh}}$ are the beating period and the beating harmonicity time, respectively. The full width at half maximum of the red curve is measured using a Gaussian fitting curve (dotted line), which gives $2 \tau_{\mathrm{bh}}=48 \mathrm{fs}$ with an uncertainty of $\Delta \tau=6 \mathrm{ps}$.

With $\bar{I}_{1}=\bar{I}_{2}$, we use Eq. (3) to obtain $\tau_{\text {bh }} \approx 4 /\left(\Delta \omega_{1}+\right.$ $\left.\Delta \omega_{2}\right) \approx 22$ ps while the beating period from Eq. (1) is $\tau_{\mathrm{b}}=$ 28 fs. For scanning $\tau$ from $-\tau_{\mathrm{bh}}$ to $\tau_{\mathrm{bh}}$, the length difference of the interferometer must be scanned over a distance of about $7 \mathrm{~mm}$ with a precision of at least $200 \mathrm{~nm}$. The scanning speed of the device does not allow the measurement to be done in a reasonably short time (demanding more than $10 \mathrm{~h}$ ). To accelerate the measurement, we scanned $\tau$ from -90 to $90 \mathrm{ps}$ in several steps separated by about $10 \mathrm{ps}$. In each step, $\tau$ was scanned over an interval of $120 \mathrm{fs}$ with a high resolution, and then the mirror was shifted by a distance of $1.5 \mathrm{~mm}$ without measurements to the next position. The measurement results are shown in Fig. 5 by the piecewise plotted green curve. The insets illustrate the curve segments measured at two steps around $\tau=-15 \mathrm{ps}$ and $\tau=25 \mathrm{ps}$. We connected the middle points of all the measured segments (average values of the signal) with straight red lines, as shown in the figure, and obtained an approximate scaled intensity correlation function. The measured beating harmonicity time is $\tau_{\mathrm{bh}}=24 \pm 3 \mathrm{ps}$ (see Fig. 5) that matches well 22 ps obtained in the calculation. Also for beams with Poissonian statistics, the agreement between the theory and measurements is good. The results support the previous remark that Eq. (3) holds for Poissonian statistics as well.

\section{B. Polarization-ring thickness}

Next, we verify our predictions on the dependence of the polarization-ring thickness $\sigma$ given by Eq. (4) on the statistics of the interfering beams. In the experiments, two independent light beams with orthogonal polarizations were combined into a single beam, using a polarizing beam splitter, and sent directly to the two-photon detector. Let the beams be $x$ and 


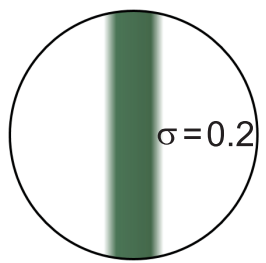

(a)

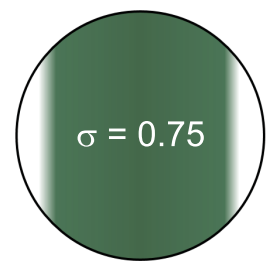

(b)

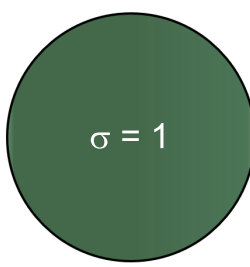

(c)
FIG. 6. Side view of the polarization ring (green) subtended by the Poincaré vector on the Poincaré sphere for (a) $\sigma=0.2$, (b) $\sigma=$ 0.75 , and (c) $\sigma=1$.

$y$ polarized having the instantaneous intensities $I_{x}$ and $I_{y}$, respectively. Since the beams have orthogonal polarizations and different wavelengths, their focal spots do not overlap perfectly at the entrance of the photomultiplier tube. Therefore, the signal we measure is

$$
J=A\left[\left\langle I_{x}^{2}\right\rangle+\left\langle I_{y}^{2}\right\rangle+a\left\langle I_{x} I_{y}\right\rangle\right],
$$

where $a$ is an unknown coefficient that takes into account the imperfect overlap of the focal spots. By blocking one of the beams at a time, we measure the signals $J_{x}=A\left\langle I_{x}^{2}\right\rangle$ and $J_{y}=A\left\langle I_{y}^{2}\right\rangle$. Since $\left\langle I_{x} I_{y}\right\rangle=\left(J-J_{x}-J_{y}\right) /(A a)$, we use Eq. (4) to obtain

$$
\sigma=\sqrt{3 \frac{\left(J_{x}+J_{y}\right)(a+2)-2 J}{\left(J_{x}+J_{y}\right)(a-2)+2 J}} .
$$

In total, 34 measurements per quantity are done in each experiment. In the first experiment we use two Poissonian singlefrequency Thorlabs' lasers L1550P5DFB ( $x$ polarized, $\lambda_{1}=$ $1550 \mathrm{~nm}$ ) and L1310P5DFB ( $y$ polarized, $\lambda_{2}=1310 \mathrm{~nm}$ ), both with $0.5 \mathrm{~mW}$ power, and measure the parameter $a$. To ensure that the beam powers are equal, we tune them until $J_{x} \approx J_{y}$. In the experiments, the monitored signals are $J_{x}=$ $4596 \pm 62$ and $J_{y}=4611 \pm 72$, where 62 and 72 are standard deviations. Then we measure $J=11837 \pm 155$, and requiring $\sigma=0$, which holds for independent beams with Poissonian statistics [see below Eq. (4)], obtain $a=0.57 \pm 0.01$ from Eq. (7). We repeat the measurement by changing the power levels of the lasers and obtain $\sigma$ values close to 0 . As an example, in an experiment at a low power $(0.2 \mathrm{~mW})$, we obtained $J_{x}=918 \pm 32, J_{y}=895 \pm 32$, and $J=2315 \pm 50$, from which it follows that $\sigma=0.2 \pm 0.2$. We note that at this power level, the lasers already operate close to the laser threshold conditions, at which the intensity fluctuations are more pronounced. The thickness of the green ring on the surface of the Poincare sphere in Fig. 6(a) corresponds to $\sigma=0.2$.

In the next experiment, we replace the diode L1550P5DFB with an $x$-polarized multimode laser diode L1480G1 $\left(\lambda_{1}=\right.$
$1480 \mathrm{~nm}$ ) that obeys Gaussian statistics. Since the new wavelength is close to the previous one and we do not change the setup, the parameter $a$ stays approximately the same. As an example, at a power level of $0.3 \mathrm{~mW}$ for the beams, we measured $J_{x}=2960 \pm 60, J_{y}=1483 \pm 39$, and $J=5316 \pm$ 73. Insertion of these values into Eq. (7) gives $\sigma=0.7 \pm 0.1$. In another measurement, we changed the power levels and obtained $J_{x}=4051 \pm 92, J_{y}=2147 \pm 48$, and $J=7301 \pm$ 106 , from which it follows that $\sigma=0.8 \pm 0.1$. Figure 6(b) shows a green ring with a thickness of 0.75 on the Poincaré sphere. The measured values of $\sigma$ are in good agreement with the theoretical prediction of $\sigma=\sqrt{3 / 5} \approx 0.77$ for two fields with different, Gaussian and Poissonian, statistics.

To complete the experiments, we also measured $\sigma$ for light composed of two beams with Gaussian statistics, in which case $\sigma=1$ must hold. In the setup, we used the same $x$ polarized multimode diode L1480G1 as in the above example and a new, fiber-coupled broadband Agilent 83438A erbium ASE source (the center wavelength is $1550 \mathrm{~nm}$ ) with the output polarized in the $y$ direction. The center wavelengths of the sources are closer to each other than in the above examples, resulting in an improved overlap of the laser spots at the entrance of the photodetector. We remeasured the parameter $a$, using a Poissonian fiber-coupled single-frequency laser at a $1550 \mathrm{~nm}$ wavelength (Agilent HP 81689A) instead of the ASE source and requiring that $\sigma=\sqrt{3 / 5}$ for equal laser powers. In this measurement, we obtained $J_{x}=1070 \pm 36, J_{y}=502 \pm$ 24 , and $J=2104 \pm 34$, which implied $a=1.01 \pm 0.09$. Replacing the Poissonian source with the Gaussian one and setting the power levels at the detector to be approximately $0.1 \mathrm{~mW}$, we measured $J_{x}=659 \pm 26, J_{y}=615 \pm 28$, and $J=1597 \pm 44$. Using Eq. (7) we then obtained $\sigma=1.0 \pm$ 0.1 [see Fig. 6(c)], thus verifying the prediction of equally probable instantaneous polarization states for such fields.

\section{CONCLUSIONS}

In this Rapid Communication, we measured the beating harmonicity times and the polarization-ring thicknesses on the Poincaré sphere for several superpositions of independent light beams with different center frequencies. The obtained experimental results matched well to the theoretically obtained values not only for beams with Gaussian statistics, for which the theory was originally formulated, but also when one or both of the beams obey Poissonian statistics. We expect that the results offer fundamental insights into the polarimetric properties of light and electromagnetic interference.

\section{ACKNOWLEDGMENTS}

This work was supported by the Academy of Finland (308394, 308393, and 320166). The authors thank Prof. Matthieu Roussey for his help in conducting the experiments.
[1] C. Brosseau, Fundamentals of Polarized Light: A Statistical Optics Approach (Wiley, Hoboken, NJ, 1998).
[2] J. J. Gil and R. Ossikovski, Polarized Light and the Mueller Matrix Approach (CRC, Boca Raton, FL, 2016). 
[3] B. E. A. Saleh and M. C. Teich, Fundamentals of Photonics, 2nd ed. (Wiley, Hoboken, NJ, 2007).

[4] E. Hecht, Optics, 5th ed. (Pearson, London, 2017).

[5] A. Norrman, A. T. Friberg, J. J. Gil, and T. Setälä, Dimensionality of random light fields, J. Eur. Opt. Soc. Rapid Publ. 13, 36 (2017).

[6] J. J. Gil, A. Norrman, A. T. Friberg, and T. Setälä, Intensity and spin anisotropy of three-dimensional polarization states, Opt. Lett. 44, 3578 (2019).

[7] A. Norrman, J. J. Gil, A. T. Friberg, and T. Setälä, Polarimetric nonregularity of evanescent waves, Opt. Lett. 44, 215 (2019).

[8] S. Hooker and C. Webb, Laser Physics (Oxford University Press, Oxford, U.K., 2010).

[9] A. F. Fercher, W. Drexler, C. K. Hitzenberger, and T. Lasser, Optical coherence tomography-principles and applications, Rep. Prog. Phys. 66, 239 (2003).

[10] W. Demtröder, Laser Spectroscopy: Basic Concepts and Instrumentation (Springer, Berlin, 2003).

[11] K. G. Gåsvik, Optical Metrology (Wiley, Hoboken, NJ, 2002).
[12] O. Korotkova, Random Light Beams: Theory and Applications (CRC, Boca Raton, FL, 2014).

[13] A. T. Friberg and T. Setälä, Electromagnetic theory of optical coherence [Invited], J. Opt. Soc. Am. A 33, 2431 (2016).

[14] A. Shevchenko and T. Setälä, Interference and polarization beating of independent arbitrarily polarized polychromatic optical waves, Phys. Rev. A 100, 023842 (2019).

[15] F. Boitier, A. Godard, E. Rosencher, and C. Fabre, Measuring photon bunching at ultrashort timescale by two-photon absorption in semiconductors, Nat. Phys. 5, 267 (2009).

[16] A. Shevchenko, M. Roussey, A. T. Friberg, and T. Setälä, U1trashort coherence times in partially polarized stationary optical beams measured by two-photon absorption, Opt. Express 23, 31274 (2015).

[17] A. Shevchenko, M. Roussey, A. T. Friberg, and T. Setälä, Polarization time of unpolarized light, Optica 1, 64 (2017).

[18] L. Mandel and E. Wolf, Optical Coherence and Quantum Optics (Cambridge University Press, Cambridge, U.K., 1995). 